\title{
VISCOSITY SOLUTIONS FOR DYNAMIC PROBLEMS WITH SLIP-RATE DEPENDENT FRICTION
}

\author{
BY \\ IOAN R. IONESCU \\ Laboratoire de Mathématiques, CNRS and Université de Savoie, Campus Scientifique, 73376 Le \\ Bourget-du-Lac Cedex, France
}

\begin{abstract}
The dynamic evolution of an elastic medium undergoing frictional slip is considered. The Coulomb law modeling the contact uses a friction coefficient that is a non-monotone function of the slip-rate. This problem is ill-posed, the solution is nonunique and shocks may be created on the contact interface. In the particular case of the one-dimensional shearing of an elastic slab, the (perfect) delay convention can be used to select a unique solution. Different solutions in acceleration and deceleration processes are obtained. To transform the ill-posed problem into a well-posed one and to justify the choice of the perfect delay criterion, a visco-elastic constitutive law with a small viscosity is used here. An existence and uniqueness result is obtained in three dimensions. The assumptions on the functions implied in the contact model are weak enough to include both the normal compliance and the Tresca model. The following conjecture, based on results of numerical simulations, is stated: in the elastic case, the solution chosen by the perfect delay convention is the one obtained from the solutions of the problem with viscosity, when the viscosity tends to zero.
\end{abstract}

1. Introduction. The first existence and uniqueness results in the mathematical approach of the dynamic contact problems with friction in elasticity were obtained by Duvaut and Lions [2]. They studied the special case of a prescribed normal pressure where the contact surface is known in advance. To obtain some existence results without this restrictive assumption, the non-penetrability of mass was relaxed by Martins and Oden [12] (see also [9] for a more general result) by considering the normal compliance model of contact with friction.

Since the pure elastic problem with friction seems to be very irregular, they considered only the viscous case. As far as we know, there exists no general existence result for dynamic elastic contact.

All of the above results involve a fixed friction coefficient $\mu$. In the study of many frictional processes (stick-slip motions, earthquakes modeling, etc.), the friction coefficient has to be considered variable during the slip. A current choice of such a variation

Received May 31, 2000.

2000 Mathematics Subject Classification. Primary 35L85; Secondary 74H10, 74H20, 74H25. 
is the friction law in which the cocfficient of friction is dependent on the slip rate $v_{T}$, i.e., $\mu=\mu\left(\left|v_{T}\right|\right)$. The simplest dependence of $\mu$ on the slip rate is the discontinuous jump from a "static" value (for $\left|v_{T}\right|=0$ ) down to a "dynamic" or "kinetic" value (for $\left.\left|v_{T}\right| \neq 0\right)$. The same frictional instabilities can occur when the coefficient of friction $\mu$ is a smooth and decreasing function of the slip rate (see [13] and [20].) This model of friction was studied by Ionescu and Paumier [5], [6] for the one-dimensional shearing problem of an infinite elastic slab. They pointed out that the solution of the problem is not uniquely determined. However, since the problem is ill-posed, a criterion to select the most appropriate solution with a physical interpretation is needed. Whatever is the selection rule to choose the solution, shocks will occur. A possible choice for this criterion is the so-called perfect delay convention: the system only jumps when it has no other choice (see [6] and [1]). In this way, different paths of solutions are obtained in acceleration and deceleration processes and a hysteresis phenomenon occurs.

The perfect delay convention is not related to a simple energy criterion. It comes from catastrophe theory (see, for instance, [15]) and is implicitly present in the analysis of many physical problems. It is used in the study of static or quasi-static problems (see, for instance, [7] for a slip-dependent model of friction), and it is justified by a dynamic stability analysis. That is, the static (or quasi-static) position chosen by the perfect delay convention is always stable. The use of this criterion in this context, even if it is intuitively reasonable, has no justification because the problem is already fully dynamic. However, the perfect delay convention does not work in other more realistic cases (2-D or 3-D problems).

Three techniques that can be used to transform this ill-posed problem into a wellposed one and to justify the choice of the perfect delay criterion are currently under investigation. In all three cases, another (disturbed) problem (which depends on a small parameter) is considered. This new problem is well-posed and its unique solution converges to the solution of the initial (non-disturbed, ill-posed) problem chosen by the perfect delay convention. The first method, due to Renard [17], [18], consists in adding a small mass concentrated on the friction surface. Using stability arguments, Renard has proved the convergence of the solution for a large class of loadings in the one-dimensional case. The second one uses a rate and state dependent friction law of Dieterich and Ruina type (see, for instance, [19], [16], [3] and [14]). Favreau et al. [4] have showed numerically that the limit solution, when the characteristic slip $L \rightarrow 0$ is the one corresponding to the rate dependent model, assuming the perfect delay convention. For both methods there are no existence or uniqueness results in two or three dimensions.

The third technique, which is presented in this paper, uses a visco-elastic constitutive law with small viscosity. An existence and uniqueness result is obtained in the general three-dimensional case. The proof, based on the Galerkin method, is constructive. The assumptions on the functions used in modeling the contact are weak enough to include both the normal compliance and the Tresca model. A similar result was obtained recently by Kuttler and Schillor [11]. There, an abstract existence and uniqueness theorem has been used. Concerning the existence result, their assumptions (which include the normal compliance model) are near to the ones used here. For uniqueness they considered the particular case of a bounded tangential stress. Other existence results for the dynamic 
contact with a slip-rate dependent friction were obtained by Kuttler et al. [10] for the beam problem. Note that the local existence results obtained by Jarusek and Eck [8] cannot be used in this context. Indeed, global uniqueness and existence results are needed for the regularized viscous problem.

Finally, I give here some numerical simulations for the one-dimensional problem, which show that the viscous solution converges, for a vanishing viscosity, to the perfect delay solution of the elastic problem. Based on these results, the following conjecture can be stated: in the elastic case, the solution chosen by the perfect delay convention is the one obtained from the solutions of the problem with viscosity, when the viscosity tends to zero. In this way, the perfect delay convention takes on a physical sense because it leads to a solution that is the limit of a regular (viscous) problem.

2. The one-dimensional problem without viscosity. For the convenience of the reader, we shall recall in this section the principal results obtained by Ionescu and Paumier [6] in the one-dimensional elastic case.

Let us denote by $\Omega=] 0, H[$ and consider the following initial and boundary value problem: find $v, \tau \in[0, T] \times \Omega \rightarrow \mathbf{R}$ such that

$$
\begin{aligned}
\rho \dot{v}(t, x) & =\partial_{x} \tau(t, x), \\
\dot{\tau}(t, x) & =G \partial_{x}(t, x),
\end{aligned}
$$

for all $(t, x) \in] 0, T] \times \Omega$,

$$
\begin{gathered}
v(t, H)=V \\
|\tau(t, 0)| \leq \mu(0) S \quad \text { if } v(t, 0)=0 \\
\tau(t, 0)=S \mu(|v(t, 0)|) \frac{v(t, 0)}{|v(t, 0)|}, \quad \text { if } v(t, 0) \neq 0
\end{gathered}
$$

for all $t \in] 0, T]$,

$$
v(0, x)=v_{0}(x), \quad \tau(0, x)=\tau_{0}(x) \text { for all } x \in \Omega,
$$

where $\rho, S, G>0$ are positive constants and $v_{0}, \tau_{0}$ are the initial data.

The above problem represents the one-dimensional dynamic shearing of an infinite linear elastic slab that is in frictional contact with a rigid body. The slab has the Lamé coefficients $\lambda$ and $G$, and it is bounded by the planes $x=0$ and $x=H$. On the plane $x=0$, the slab is in frictional contact with a rigid foundation. At $x=H$, the slab is dragged with a tangential velocity $V$ in the $y$-direction and it is pushed in the $x$-direction with a uniform normal stress $-S$. We assume that the displacement $u_{z}$ vanishes in the $z$-direction and $u_{x}=-\frac{S}{\lambda+2 G} x$. We denote by $u=u(t, x)$ the horizontal displacement $\left(u_{y}\right)$, by $v(t, x)=\dot{u}(t, x)$ the velocity, and by $\tau(t, x)=G \partial_{x} u(t, x)$ the shear stress. On the friction boundary $(x=0)$, the normal stress is $-S$, the tangential stress is $-\tau(t, 0)$, and (4), (5) represent a friction law with slip-rate dependent friction coefficient $\mu=\mu(v)$.

For the sake of simplicity, and without any loss of generality, we will suppose that $T \leq T^{*}=H / c$, where $c=\sqrt{\frac{G}{\rho}}$ is the wave velocity. Since $\sqrt{\rho G} v(t, x)+\tau(t, x)$ is 
constant on the characteristic lines $D_{k}=\left\{(x, t) \in \mathbf{R}^{2} ; x+c t=k\right\}$, then

$$
\sqrt{\rho G} v(t, 0)+\tau(t, 0)=S \beta(t),
$$

where $\beta:[0, T] \rightarrow \mathbf{R}$ is given by the initial conditions:

$$
\beta(t)=\frac{1}{S}\left[\tau_{0}(c t)+\sqrt{\rho G} v_{0}(c t)\right]
$$

An important role in our analysis is played by the function $g: \mathbf{R}_{+} \rightarrow \mathbf{R}$ given by

$$
g(s)=\mu(s)+a s, \quad \forall s \in \mathbf{R}_{+},
$$

where $\mu$ is the friction coefficient, which is supposed to be positive and continuous, and $a=\frac{\sqrt{\rho G}}{S}$. From (7) and (3) $(6)$, one can deduce an equivalence between the hyperbolic problem (1)-(6) and a scalar equation to be solved for all $t \in[0, T]$ that gives the boundary data $\alpha$ for a standard mixed hyperbolic problem. Indeed, $(v, \tau)$ is a (weak) solution of the problem (1)-(6) iff $\alpha:[0, T] \rightarrow \mathbf{R}$ is a piecewise continuous solution of the following family of scalar equations:

$$
(\alpha(t), \beta(t)) \in K \quad \text { a.e. } t \in[0, T],
$$

and $(v, \tau)$ is the (weak) solution of $(1)-(3),(6)$ and $v(t, 0)=\alpha(t)$, a.e. $t \in[0, T]$. Here $K$ is the graph of the odd extension of $g$, i.e.,

$$
K=:\{0\} \times[-g(0), g(0)] \cup\{(x, g(x)) ; x>0\} \cup\{(x,-g(-x)) ; x<0\} .
$$

Two different qualitative behaviors of the solution are found, depending on the monotonicity of $g$.

In the first case, $g$ is increasing (i.e., $\mu^{\prime}(s)>-a$ ), and there is a unique continuous solution $t \rightarrow \alpha(t)=v(t, 0)$ for all continuous $\beta$. Hence, there exists a unique (weak) solution $(v, \tau)$ of the problem (1)-(6) and the mapping that associates the solution $(v, \tau)$ to every data $\left(V, v_{0}, \tau_{0}\right)$ is continuous.

In the second case, called the "irregular behavior", $g$ is not increasing (see Fig. 1). This is the case when the friction coefficient $\mu$ has a "large" enough weakening rate $-\mu$ ' (when it decreases from the static value $\mu_{s}$ to the dynamic one $\mu_{d}$ ). For instance, let us suppose that there exists $\alpha_{1}>0$, such that $g$ is increasing on $\left[\alpha_{1},+\infty\right.$ [ and decreasing on $\left[0, \alpha_{1}\right]$ and $\beta$ is monotone and continuous. Then we find that, for all weak solutions $(v, \tau)$ of $(1)-(6)$ and all $t \in[0, T]$, the point $(v(t, 0), \beta(t))$ belongs to the solid line $K$ plotted in Fig. 1. The solution $v(t, 0)=\alpha(t)$ is unique if $\beta(t)<g\left(\alpha_{1}\right)$ or $g(0)<\beta(t)$, but there are two or three solutions for $g\left(\alpha_{1}\right) \leq \beta(t) \leq g(0)$. Hence at each time $t$ we have to choose between these three solutions.

However, since the solution of the problem (1)-(6) or (9) is not uniquely determined, we need a criterion to select the most appropriate solution with a physical interpretation.

Let us remark firstly that whatever selection rule is chosen, shocks will occur. Indeed, if $\beta\left(t_{1}\right)<g\left(\alpha_{1}\right)$ and $\beta\left(t_{2}\right)>g(0)$, then the functions $t \rightarrow \alpha(t)=v(t, 0)$ and $t \rightarrow \tau(t, 0)$ must have at least one discontinuity in the time interval $\left[t_{1}, t_{2}\right]$ if $\beta$ is increasing. In conclusion, there exist no continuous solutions, even if the data are continuous (i.e., all the regularity and compatibility assumptions are satisfied).

A possible choice of a criterion to select a solution is the (perfect) delay convention: the system only jumps when it has no other choice (see Ionescu and Paumier [6], Campillo 


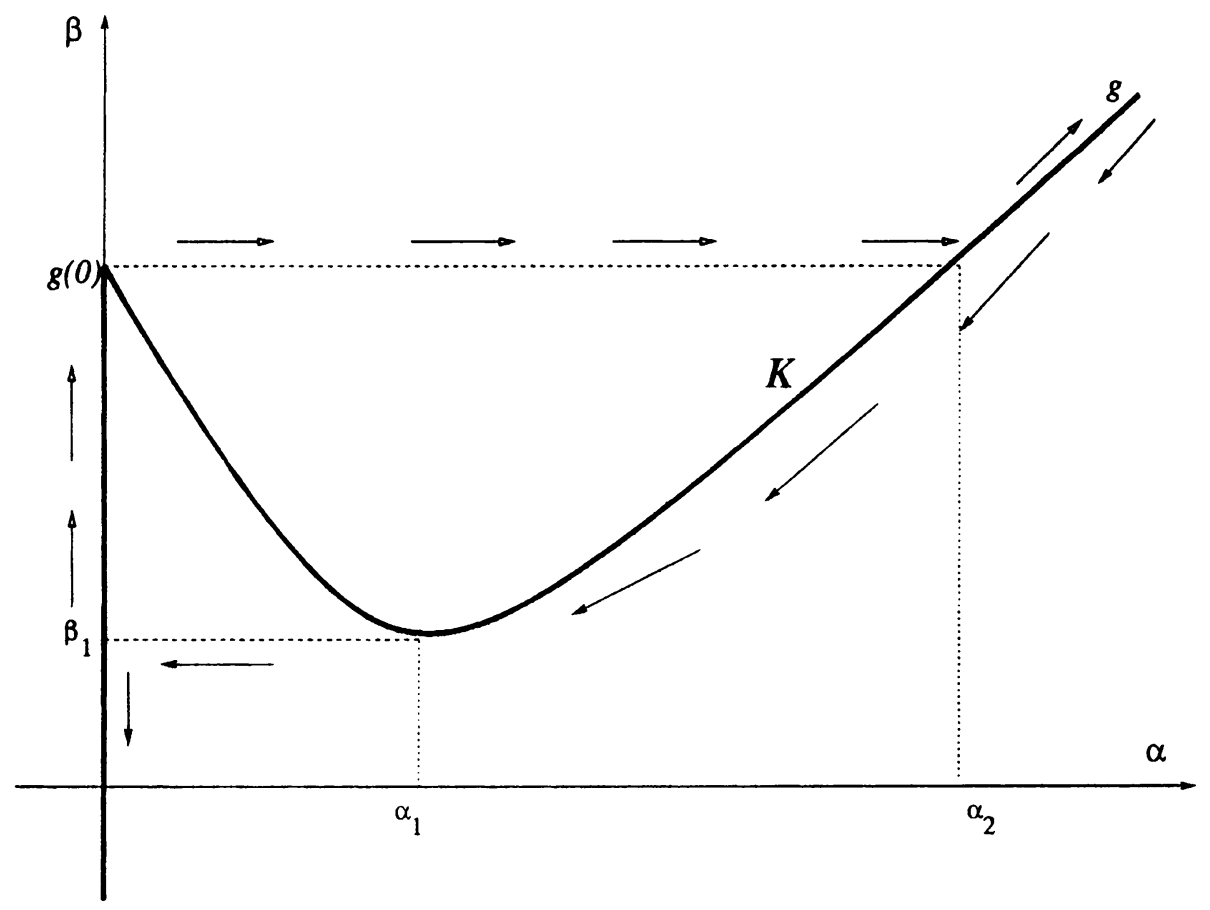

Fig. 1. The set $K$ (solid line) of the function $g$ in the irregular case. At each time $t$ for which $\beta(t) \in] \beta_{1}, g(0)$ [ the problem (9) has three solutions. The arrows show two paths of solutions selected with the perfect delay convention in acceleration ( $\beta$ increasing) and deceleration ( $\beta$ decreasing) processes. Note a hysteresis phenomenon.

et al. [1]). Hence the physical solution $v(t, 0)$ maximizes its interval of continuity. In this way (see Fig. 1), different paths of solutions are obtained in acceleration ( $\beta$ increasing) and deceleration ( $\beta$ decreasing) processes and a hysteresis phenomenon occurs. One consequence is that $v(t, 0)$ never follows the decreasing branch where $\mu^{\prime}(v(t, 0))<-a$ (i.e., $v(t, 0) \notin] \alpha_{1}, \alpha_{2}[$ ).

It was shown in Ionescu and Paumier [5], [6] that the perfect delay convention is not related to a simple energy criterion. One may notice that the delay criterion, which comes from catastrophe theory (see, for instance, Poston and Stewart [15]), is implicitly present in the analysis of many physical problems. Generally speaking, it is used in the study of static or quasi-static problems (see Ionescu and Paumier [7]), and it is justified by a dynamic stability analysis, that is, the (static or quasi-static) position chosen by the perfect delay convention is always a stable position. The use of this criterion in our context, even if it is intuitively reasonable, has no justification because our problem is already fully dynamic. We will see in the last section that this choice can be motivated by the analysis of a regularized viscous problem.

3. Statement of the viscous problem. Let $\Omega \subset \mathbf{R}^{N}(N=2,3)$ be a bounded domain, representing the interior of a viscoelastic body, with a smooth boundary $\Gamma=\partial \Omega$, 
which is divided into three disjoint parts $\Gamma=\bar{\Gamma}_{d} \cup \bar{\Gamma}_{c} \cup \bar{\Gamma}_{f}$ with meas $\left(\Gamma_{d}\right)>0$. The mechanical problem (MP) consists in finding the displacement field $u:[0, T] \times \Omega \rightarrow \mathbf{R}^{N}$ such that

$$
\begin{gathered}
\sigma(t)=\mathcal{A} \epsilon(u(t))+\eta \mathcal{C} \epsilon(\dot{u}(t)), \quad \rho \ddot{u}(t)=\operatorname{div} \sigma(t)+b(t) \quad \text { in } \Omega, \\
u(t)=0 \quad \text { on } \Gamma_{d}, \quad \sigma(t) n=l(t) \quad \text { on } \Gamma_{c}, \\
\sigma_{n}(t)=-h_{n}\left(u_{n}^{+}(t)\right) \quad \text { on } \Gamma_{f}, \\
\sigma_{T}(t)=-h_{T}\left(u_{n}^{+}(t)\right) \mu\left(\left|\dot{u}_{T}(t)\right|\right) \frac{\dot{u}_{T}(t)}{\left|\dot{u}_{T}(t)\right|} \quad \text { if } \dot{u}_{T}(t) \neq 0 \text { on } \Gamma_{f}, \\
\left|\sigma_{T}(t)\right| \leq h_{T}\left(u_{n}^{+}(t)\right) \mu(0) \quad \text { if } \dot{u}_{T}(t)=0 \text { on } \Gamma_{f}, \\
u(0)=u_{0}, \quad \dot{u}(0)=v_{0} \quad \text { in } \Omega,
\end{gathered}
$$

where $\eta>0$ is a viscosity coefficient, $\rho>0$ is the density, $\mathcal{A}, \mathcal{C}$ are the fourth-order tensors, $\sigma$ is the stress tensor, $\epsilon(u)=\frac{1}{2}\left(\nabla u+\nabla^{T} u\right)$ is the small strain tensor, $n$ is the unit outward normal vector on $\Gamma, \sigma_{n}=\sigma n \cdot n$ is the normal stress, $\sigma_{T}=\sigma n-\sigma_{n} n$ is the tangential stress, $u_{n}=u \cdot n$ is the normal displacement, $u_{n}^{+}$is its positive part, and $u_{T}=u-u_{n} n$ is the tangential displacement. Here $b$ represents the given body forces and $l$ is the load on $\Gamma_{c}$.

Equations (12)-(14) represent the contact with friction along a candidate surface $\Gamma_{f}$ with a rigid and fixed body. If there exists a normal gap $g_{\text {gap }}$ between the viscoelastic body and the foundation, measured in the undeformed configuration, then $u_{n}^{+}$have to be replaced by $\left(u_{n}-g_{\mathrm{gap}}\right)^{+}$.

In (12) the normal stress $\sigma_{n}$ is a function of the penetration $u_{n}^{+}$. Two cases are often used in the literature. In the first one, the Tresca model, the normal stress is given, i.e., $h_{n}(v)=S_{n}$; hence, the contact surface is also given. The second one is the normal compliance model characterized by a power-law relationship, i.e., $h_{n}(v)=|v|^{m_{n}}, h_{T}(v)=$ $|v|^{m_{T}}$.

Equations (13), (14) assert that if there is contact, the tangential (friction) stress is bounded by a function of penetration $u_{n}^{+}$multiplied by the static value of the "friction coefficient" $\mu(0)$. If such a limit is not attained, then sliding does not occur. Otherwise the friction stress is opposite to the slip rate and its absolute value depends on the slip velocity. As a matter of fact, if we put $h_{T}(v)=r\left(h_{n}(v)\right) h_{n}(v)$, then we get that $\nu=\frac{\left|\sigma_{T}\right|}{\left|\sigma_{n}\right|}=r\left(\left|\sigma_{n}\right|\right) \mu\left(\left|\dot{u}_{T}\right|\right)$, which corresponds with a generalization of Coulomb's friction law. Indeed, in this case, the coefficient of friction $\nu$ is no longer constant, and it accommodates the dependence on the normal stress and on the slip rate.

4. Assumptions, notation and preliminaries. In the study of the problem (10)(15), the following assumptions are used: $\mathcal{A}, \mathcal{C}$ are symmetric and positively defined fourth-order tensors, i.e.,

$$
\begin{gathered}
\mathcal{A}_{i j k l}, \mathcal{C}_{i j k l} \in L^{\infty}(\Omega), \quad \mathcal{A}(x) \epsilon: \sigma=\mathcal{A}(x) \sigma: \epsilon, \quad \mathcal{C}(x) \epsilon: \sigma=\mathcal{C}(x) \sigma: \epsilon, \\
\exists \alpha>0 \text { such that } \mathcal{A}(x) \epsilon: \epsilon \geq \alpha|\epsilon|^{2}, \quad \mathcal{C}(x) \epsilon: \epsilon \geq \alpha|\epsilon|^{2},
\end{gathered}
$$

a.e. $x \in \Omega, \forall i, j, k, l=\overline{1, N}$ and for all $\sigma, \epsilon \in \mathbf{R}_{S}^{N \times N}$. 
The friction coefficient $\mu: \Gamma_{f} \times \mathbf{R}_{+} \rightarrow \mathbf{R}_{+}$has the following properties:

$$
\begin{gathered}
\left|\mu\left(x, u_{1}\right)-\mu\left(x, u_{2}\right)\right| \leq L\left|u_{1}-u_{2}\right| \quad \forall u_{1}, u_{2} \in\left[0,+\infty \mid \text { a.e. } x \in \Gamma_{f},\right. \\
0 \leq \mu(x, u) \leq \mu_{0} \quad \text { a.e. } x \in \Gamma_{f}, \quad \forall u \in \mathbf{R}_{+},
\end{gathered}
$$

and the function $x \rightarrow \mu(x, u)$ is measurable for all $u \in \mathbf{R}_{+}$. Concerning the functions $h_{n}$ and $h_{T}$, we shall suppose that $x \rightarrow h_{i}(x, u)$ is measurable for all $u \in \mathbf{R}_{+}$and that there exist $C_{i}, D_{i}, E_{i} \geq 0$ and $m_{i} \geq 1$ such that

$$
\begin{gathered}
\left|h_{i}(x, u)\right| \leq C_{i}+D_{i}|u|^{m_{i}} \\
\left|h_{i}\left(x, u_{1}\right)-h_{i}\left(x, u_{2}\right)\right| \leq E_{i}\left(1+\left|u_{1}\right|^{m_{i}-1}+\left|u_{2}\right|^{m_{i}-1}\right)\left|u_{1}-u_{2}\right|,
\end{gathered}
$$

a.e. $x \in \Gamma_{f}$, and for all $u, u_{1}, u_{2} \in \mathbf{R}_{+}$, with $i=n$ or $i=T$. We denote by $p_{n}=$ $m_{n}+1, p_{T}=m_{T}+1, p=\max \left\{p_{n}, p_{T}\right\}$ and let us suppose that

$$
p<3 \text { if } N=3 \text {. }
$$

Let $S_{i}(x)=h_{i}(x, 0)$, and let us denote by

$$
H_{i}(x, u)=\int_{0}^{u}\left[h_{i}(x, v)-S_{i}(x)\right] d v,
$$

a.e. $x \in \Gamma_{f}$, and for all $u \in \mathbf{R}_{+}$, and let us suppose that $H_{i}(x, u) \geq 0$ a.e. $x \in \Gamma_{f}$, with $i=n$ or $i=T$. We shall also suppose that the density $\rho \in L^{\infty}(\Omega)$ is positive, i.e., there exists $\rho_{0}$ such that $\rho(x) \geq \rho_{0}>0$. Finally, the load $l$ and the body forces $b$ will be supposed to have the regularity

$$
l \in W^{1,2}\left(0, T,\left[L^{2}\left(\Gamma_{c}\right)\right]^{N}\right), \quad b \in W^{1,2}\left(0, T,\left[L^{2}(\Omega)\right]^{N}\right) .
$$

Let us denote by $H:=\left[L^{2}(\Omega)\right]^{N}$ endowed with the inner product

$$
(u, v):=\int_{\Omega} \rho u \cdot v d x, \quad \forall u, v \in H
$$

which generates an equivalent norm denoted by $|\cdot|$. Let $V$ be the closed subspace of $\left[H^{1}(\Omega)\right]^{N}$ given by

$$
V:=\left\{v \in\left[H^{1}(\Omega)\right]^{N} ; v=0 \text { on } \Gamma_{d}\right\}
$$

and let us suppose that

$$
u_{0}, v_{0} \in V \text {. }
$$

If we denote by $a, c: V \times V \rightarrow \mathbf{R}$ the following bilinear and symmetric applications

$$
a(u, v):=\int_{\Omega} \mathcal{A} \epsilon(u): \epsilon(v), \quad c(u, v):=\int_{\Omega} \mathcal{C} \epsilon(u): \epsilon(v), \quad \forall u, v \in V,
$$

then from (16) we find $M>0$ such that

$$
|a(u, v)| \leq M\|u\|_{V}\|v\|_{V}, \quad|c(u, v)| \leq M\|u\|_{V}\|v\|_{V}, \quad \forall u, v \in V .
$$

From (17) and the Korn inequality, we deduce that there exists $d>0$ such that

$$
a(v, v) \geq d\|v\|_{V}^{2}, \quad c(v, v) \geq d\|v\|_{V}^{2} \quad \forall v \in V .
$$


Finally, we define $f \in W^{1,2}\left(0, T, V^{\prime}\right)$ and $j: V \times V \times V \rightarrow \mathbf{R}$ as follows:

$$
\begin{gathered}
\langle f(t), v\rangle=: \int_{\Omega} b(t) \cdot v d x+\int_{\Gamma_{c}} l(t) \cdot v d s, \\
j(u, v, w):=\int_{\Gamma_{f}} h_{T}\left(u_{n}^{+}\right) \mu\left(\left|v_{T}\right|\right)\left|w_{T}\right| d s+\int_{\Gamma_{f}} h_{n}\left(u_{n}^{+}\right) w_{n} d s, \quad \forall u, v, w \in V .
\end{gathered}
$$

Using this notation, one can easily deduce that any solution of (10)-(15) satisfies the following variational problem (VP): find $u:[0, T] \rightarrow V$ such that

$$
\begin{aligned}
(\ddot{u}(t), v-\dot{u}(t))+a(u(t), & v-\dot{u}(t))+\eta c(\dot{u}(t), v-\dot{u}(t)) \\
& +j(u(t), \dot{u}(t), v)-j(u(t), \dot{u}(t), \dot{u}(t)) \geq\langle f(t), v-\dot{u}(t)\rangle,
\end{aligned}
$$

for all $v \in V$, a.e. $t \in] 0, T[$ and

$$
u(0)=u_{0}, \quad \dot{u}(0)=v_{0} .
$$

5. Existence and uniqueness of the solution. The main result of this section is the following:

THEOREM 5.1. There exists a unique solution of (VP) with the following regularity:

$$
u \in W^{1, \infty}(0, T, V) \cap W^{2,2}(0, T, H) .
$$

A similar theorem was obtained recently by Kuttler and Schillor [11]. There, an abstract existence and uniqueness theorem has been used. Concerning the existence result, their assumptions (which include the normal compliance model) are near to the ones used here. They supposed in addition that the function $h_{n}$ is increasing and $h_{n}(0)=h_{T}(0)=0$ (which excludes the Tresca model) but the restriction (22) is removed. However, we present here the main points of a constructive proof of the existence, based on the Galerkin method. Concerning the uniqueness result, in [11] is considered the particular case of a bounded tangential stress, i.e., $h_{T}$ is supposed to be a bounded function.

The following lemma will be useful in the proof of this theorem.

Lemma 5.1. Let $\Omega \subset \mathbf{R}^{N}$ be as above and let $\alpha \in\left[2, \frac{2(N-1)}{N-2}\right]$ if $N \geq 3$ and $\alpha \geq 2$ if $N=2$. Then for $\beta=\frac{N(\alpha-2)+2}{2 \alpha}$ if $N \geq 3$ or if $N=2$ and $\alpha=2$ and for all $\left.\beta \in\right] \frac{\alpha-1}{\alpha}, 1[$ if $N=2$ and $\alpha>2$, there exists a constant $C=C(\beta)$ such that

$$
\|v\|_{L^{\alpha}(\Gamma)} \leq C\|v\|_{L^{2}(\Omega)}^{1-\beta}\|v\|_{H^{1}(\Omega)}^{\beta}, \quad \forall v \in H^{1}(\Omega)
$$

For the convenience of the reader we give here a simple proof of this lemma.

Proof. If $\alpha=\frac{2(N-1)}{N-2}$ for $N \geq 3$, then $\beta=1$ and (32) follows from the trace immersion theorem. Let us suppose now that $\alpha \in\left[2, \frac{2(N-1)}{N-2}\left[\right.\right.$ if $N \geq 3$. If $v \in H^{1}(\Omega)$, then $|v|^{\alpha}$ belongs to $W^{1,1}(\Omega)$. Using the continuity of the trace map from $W^{1,1}(\Omega)$ into $L^{1}(\Gamma)$, we deduce

$$
\begin{aligned}
\|v\|_{L^{\alpha}(\Gamma)}^{\alpha}=\left\||v|^{\alpha}\right\|_{L^{1}(\Gamma)} & \leq C\left\{\left\||v|^{\alpha}\right\|_{L^{1}(\Omega)}+\left\|\nabla|v|^{\alpha}\right\|_{L^{1}(\Omega)}\right\} \\
& \leq C\left\{\|v\|_{L^{2}(\Omega)}\|v\|_{L^{2(\alpha-1)}(\Omega)}^{\alpha-1}+\left\||v|^{\alpha-1}|\nabla v|\right\|_{L^{1}(\Omega)}\right\} .
\end{aligned}
$$


If $\alpha=2$, then (32) easily follows with $\beta=\frac{1}{2}$. For $\alpha>2$, let us denote by

$$
q=\frac{2}{\alpha(1-\beta)}, \quad r=\frac{2((\alpha-1) q-2)}{q-2} .
$$

Then we get $r=\frac{2 N}{N-2}$ for $N \geq 3$ and $r>1$ for $N=2$ and, using the immersion of $H^{1}(\Omega)$ in $L^{r}(\Omega)$, we deduce

$$
\begin{aligned}
& \|v\|_{L^{2}(\Omega)}\|v\|_{L^{2(\alpha-1)}(\Omega)}^{\alpha-1}+\left\||v|^{\alpha-1}|\nabla v|\right\|_{L^{1}(\Omega)} \\
& \quad \leq C\|v\|_{L^{2}(\Omega)}\|v\|_{H^{1}(\Omega)}^{\alpha-1}+\|\nabla v\|_{L^{2}(\Omega)}\|v\|_{L^{2}(\Omega)}^{\frac{2}{q}}\|v\|_{L^{r^{2}(\Omega)}}^{\frac{(\alpha-1) q-2}{q}} \\
& \quad \leq C\|v\|_{L^{2}(\Omega)}^{\alpha(1-\beta)}\|v\|_{H^{1}(\Omega)}^{\alpha \beta}
\end{aligned}
$$

and (32) follows from the last two inequalities.

Proof of Theorem 5.1.

Uniqueness. Let $u_{1}$ and $u_{2}$ be two solutions of (VP) and let us denote by $\bar{u}=: u_{1}-u_{2}$. If we write the variational statement, successively for $u_{1}$ and $u_{2}$, taking $v=\dot{u}_{2}(t)$ in the first inequality and $v=\dot{u}_{1}(t)$ in the second, and adding the resulting inequalities, then we obtain

$$
\begin{aligned}
& (\ddot{\bar{u}}(t) \dot{\bar{u}}(t))+a(\dot{\bar{u}}(t), \bar{u}(t))+\eta c(\dot{\bar{u}}(t), \dot{\bar{u}}(t))+\int_{\Gamma_{f}}\left[h_{n}\left(u_{1 n}^{+}(t)\right)-h_{n}\left(u_{2 n}^{+}(t)\right)\right] \dot{\bar{u}}_{n}(t) d s \\
& \quad+\int_{\Gamma_{f}}\left[h_{T}\left(u_{1 n}^{+}(t)\right) \mu\left(\left|\dot{u}_{1 T}(t)\right|\right)-h_{T}\left(u_{2 n}^{+}(t)\right) \mu\left(\left|\dot{u}_{2 T}(t)\right|\right)\right]\left[\left|\dot{u}_{1 T}(t)\right|-\left|\dot{u}_{2 T}(t)\right|\right] d s \leq 0 .
\end{aligned}
$$

Bearing in mind (18)-(21), from the above inequality we get

$$
\begin{aligned}
& \frac{1}{2} \frac{d}{d t}\left\{|\dot{\bar{u}}(t)|^{2}+a(\bar{u}(t), \bar{u}(t))\right\}+\eta c(\dot{\bar{u}}(t), \dot{\bar{u}}(t)) \\
& \leq E_{n} \int_{\Gamma_{f}}\left\{1+\left|u_{1 n}(t)\right|^{m_{n}-1}+\left|u_{2 n}(t)\right|^{m_{n}-1}\right\}\left|\bar{u}_{n}(t)\right|\left|\dot{\bar{u}}_{n}(t)\right| d s \\
& \quad+\mu_{0} E_{T} \int_{\Gamma_{f}}\left\{1+\left|u_{1 n}(t)\right|^{m_{T}-1}+\left|u_{2 n}(t)\right|^{m_{T}-1}\right\}\left|\bar{u}_{n}(t)\right|\left|\dot{\bar{u}}_{T}(t)\right| d s \\
& \quad+L \int_{\Gamma_{f}}\left(C_{T}+D_{T}\left|u_{1 n}(t)\right|^{m_{T}}\right)\left|\dot{\bar{u}}_{T}(t)\right|^{2} d s .
\end{aligned}
$$

The first two integrals from the right part of the above inequality can easily be estimated as follows:

$$
\begin{aligned}
C\{1 & \left.+\left\|u_{1}(t)\right\|_{L^{p}(\Gamma)}^{p-2}+\left\|u_{2}(t)\right\|_{L^{p}(\Gamma)}^{p-2}\right\}\|\bar{u}(t)\|_{L^{p}(\Gamma)}\|\dot{\bar{u}}(t)\|_{L^{p}(\Gamma)} \\
& \leq C\left\{1+\left\|u_{1}\right\|_{L^{\infty}(0, T, V)}^{p-2}+\left\|u_{2}\right\|_{L^{\infty}(0, T, V)}^{p-2}\right\}\|\bar{u}(t)\|_{V}\|\dot{\bar{u}}(t)\|_{V} \\
& \leq \frac{C}{\eta} a(\bar{u}(t), \bar{u}(t))+\frac{\eta}{4} c(\dot{\bar{u}}(t), \dot{\bar{u}}(t)) .
\end{aligned}
$$

Concerning the third integral, we use Lemma 5.1 with

$$
\beta=\frac{3(p-1)+2}{2(p+1)}<1 \quad \text { if } N=3
$$


and

$$
\beta \in] \frac{p}{p+1}, 1[\text { if } N=2
$$

to deduce

$$
\begin{aligned}
L \int_{\Gamma_{f}} & \left\{C_{T}+D_{T}\left|u_{1 n}(t)\right|^{m_{T}}\right\}\left|\dot{\bar{u}}_{T}(t)\right|^{2} d s \\
& \leq C\left\{1+\left\|u_{1}(t)\right\|_{L^{p+1}(\Gamma)}^{p-1}\right\}\|\dot{\bar{u}}(t)\|_{L^{p+1}(\Gamma)}^{2} \\
& \leq C\left\{1+\left\|u_{1}\right\|_{L^{\infty}(0, T, V)}^{p-1}\right\}|\dot{\bar{u}}(t)|^{2(1-\beta)}\|\overline{\bar{u}}(t)\|_{V}^{2 \beta} \\
& \leq C \eta^{-\frac{\beta}{1-\beta}}|\dot{\bar{u}}(t)|^{2}+\frac{\eta}{2} c(\dot{\bar{u}}(t), \dot{\bar{u}}(t)) .
\end{aligned}
$$

Since $\bar{u}(0)=\dot{\bar{u}}(0)=0$, the integration of (33) from 0 to $t$ and the above inequalities lead to:

$$
|\dot{\bar{u}}(t)|^{2}+a(\bar{u}(t), \bar{u}(t)) \leq C\left(\eta^{-1}+\eta^{-\frac{\beta}{1-\beta}}\right) \int_{0}^{t}\left\{|\dot{\bar{u}}(\alpha)|^{2}+a(\bar{u}(\alpha), \bar{u}(\alpha))\right\} d \alpha,
$$

and from Gronwall's lemma the uniqueness follows.

Existence. In order to prove the existence of the solution $u$ of (VP), we shall use the Faedo-Galerkin method. For this, let us consider $\phi_{i} \in V$ to be a sequence of linearly independent functions such that $V=\overline{\bigcup_{m=1}^{\infty} V_{m}}$, where $V_{m}=\operatorname{Span}\left\{\phi_{1}, \phi_{2}, \ldots, \phi_{m}\right\}$. Since $u_{0}, v_{0} \in V$, let $u_{0}^{m}, v_{0}^{m} \in V_{m}$ be such that

$$
u_{0}^{m} \rightarrow u_{0}, v_{0}^{m} \rightarrow v_{0} \quad \text { strongly in } V .
$$

We now introduce a family of variational problems in $V_{m}$, called $\left(\mathrm{VP}_{\epsilon}^{m}\right)$, depending on a real parameter $\epsilon>0$ that represents the regularized version of (VP). We will begin by proving that each problem has a unique solution $u_{\epsilon}^{m}$ for all $\epsilon>0$ and all $m \in \mathbf{N}$. Only after that, we shall prove that when $\epsilon \rightarrow 0$ and $m \rightarrow+\infty$ the limit, in an appropriate sense, of $u_{\epsilon}^{m}$ is the solution of (VP). Let us define $J_{\epsilon}: V \times V \times V \rightarrow V^{\prime}$, a family of regularized frictional functionals, depending on $\epsilon>0$ :

$$
\left\langle J_{\epsilon}(u, v, w), z\right\rangle=\int_{\Gamma_{f}} h_{T}\left(u_{n}^{+}\right) \mu\left(\left|v_{T}\right|\right) \frac{w_{T} \cdot z_{T}}{\sqrt{\left|w_{T}\right|^{2}+\epsilon^{2}}} d s+\int_{\Gamma_{f}} h_{n}\left(u_{n}^{+}\right) z_{n} d s .
$$

With this definition, we can introduce the following variational problem in $V_{m}$ with regularized friction $\left(\mathrm{VP}_{\epsilon}^{m}\right)$ : find $u_{\epsilon}^{m}:[0, T] \rightarrow V_{m}$ such that

$$
\left(\ddot{u}_{\epsilon}^{m}(t), v\right)+a\left(u_{\epsilon}^{m}(t), v\right)+\eta c\left(\dot{u}_{\epsilon}^{m}(t), v\right)+\left\langle J_{\epsilon}\left(u_{\epsilon}^{m}(t), \dot{u}_{\epsilon}^{m}(t), \dot{u}_{\epsilon}^{m}(t)\right), v\right\rangle=\langle f(t), v\rangle,
$$

for all $v \in V_{m}$, a.e. $\left.t \in\right] 0, T[$, and

$$
u_{\epsilon}^{m}(0)=u_{0}^{m}, \quad \dot{u}_{\epsilon}^{m}(0)=v_{0}^{m}
$$

for all $v \in V_{m}$, a.e. $\left.t \in\right] 0, T\left[\right.$. Since $(u, v) \rightarrow J_{\epsilon}(u, v, v)$ is a locally Lipschitz coninuous function on $V_{m} \times V_{m}$, we deduce that (36), (37) has a unique local solution $u_{\epsilon}^{m} \in C^{2}\left(\left[0, T_{\epsilon}^{m}\left[, V_{m}\right)\right.\right.$. 
If we put in (36), $v=\dot{u}_{\epsilon}^{m}(t)$ we get

$$
\begin{aligned}
\frac{1}{2} \frac{d}{d t}\left\{\left|\dot{u}_{\epsilon}^{m}(t)\right|^{2}+a\left(u_{\epsilon}^{m}(t), u_{\epsilon}^{m}(t)\right)+\int_{\Gamma_{f}} H_{n}\left(\left[u_{\epsilon}^{m}\right]_{n}^{+}(t)\right) d s\right\} & +\eta c\left(\dot{u}_{\epsilon}^{m}(t), \dot{u}_{\epsilon}^{m}(t)\right) \\
& \leq\left\langle f(t), \dot{u}_{\epsilon}^{m}(t)\right\rangle-\int_{\Gamma_{f}} S_{n}\left[\dot{u}_{\epsilon}^{m}\right]_{n}(t) d s
\end{aligned}
$$

and, after integrating from 0 to $t$, we obtain that there exist $A_{0}, B>0$ such that

$$
\left|\dot{u}_{\epsilon}^{m}(t)\right|+\frac{d}{2}\left\|u_{\epsilon}^{m}(t)\right\|_{V}^{2}+2 d \eta \int_{0}^{t}\left\|\dot{u}_{\epsilon}^{m}(s)\right\|_{V}^{2} d s \leq A_{0}+B \int_{0}^{t}\left\|u_{\epsilon}^{m}(s)\right\|_{V}^{2} d s .
$$

From Gronwall's lemma we deduce that $t \rightarrow\left(u_{\epsilon}^{m}(t), \dot{u}_{\epsilon}^{m}(t)\right)$, the solution of $(36)$ and (37), is bounded on its interval of existence; hence, $T_{\epsilon}^{m}=T$ and

$$
\begin{gathered}
u_{\epsilon}^{m} \text { is bounded in } L^{\infty}(0, T, V), \\
\dot{u}_{\epsilon}^{m} \text { is bounded in } L^{\infty}(0, T, H) \cap L^{2}(0, T, V) .
\end{gathered}
$$

If we put $v=\ddot{u}_{\epsilon}^{m}(t)$ in $(36)$, then we get

$$
\begin{aligned}
\left|\ddot{u}_{\epsilon}^{m}(t)\right|^{2}+a\left(u_{\epsilon T}^{m}(t), \ddot{u}_{\epsilon}^{m}(t)\right)+ & \frac{\eta}{2} \frac{d}{d t}\left[c\left(\dot{u}_{\epsilon}^{m}(t), \dot{u}_{\epsilon}^{m}(t)\right)\right] \\
& +\left\langle J_{\epsilon}\left(u_{\epsilon}^{m}(t), \dot{u}_{\epsilon}^{m}(t), \dot{u}_{\epsilon}^{m}(t)\right), \ddot{u}_{\epsilon}^{m}(t)\right\rangle=\left\langle f(t), \ddot{u}_{\epsilon}^{m}(t)\right\rangle .
\end{aligned}
$$

Let us denote by $M_{\epsilon}: \Gamma_{f} \times \mathbf{R}^{N} \rightarrow \mathbf{R}$ as follows:

$$
M_{\epsilon}(x, v)=\frac{1}{2} \int_{0}^{\left|v_{T}\right|^{2}} \frac{\mu(x, \sqrt{s})}{\sqrt{s+\epsilon^{2}}} d s
$$

and let us remark that

$$
\begin{aligned}
& \left\langle J_{\epsilon}\left(u_{\epsilon}^{m}(t), \dot{u}_{\epsilon}^{m}(t), \dot{u}_{\epsilon}^{m}(t)\right), \ddot{u}_{\epsilon}^{m}(t)\right\rangle \\
& =\int_{\Gamma_{f}} h_{T}\left(\left[u_{\epsilon}^{m}\right]_{n}^{+}(t)\right) \frac{d}{d t} M_{\epsilon}\left(\dot{u}_{\epsilon}^{m}(t)\right) d s+\int_{\Gamma_{f}} h_{n}\left(\left[u_{\epsilon}^{m}\right]_{n}^{+}(t)\right) \frac{d}{d t}\left[\dot{u}_{\epsilon}^{m}\right]_{n}(t) d s .
\end{aligned}
$$

If we integrate (40) from 0 to $t$ and we have in mind that $t \rightarrow h_{i}\left(\left[u_{\epsilon}^{m}\right]_{n}^{+}(t)\right) \in L^{2}\left(\Gamma_{f}\right)$ are absolutely continuous functions $(i=n$ or $i=T)$, then we obtain

$$
\begin{aligned}
& \int_{0}^{t}\left|\ddot{u}_{\epsilon}^{m}(\alpha)\right|^{2} d \alpha+\frac{\eta}{2} c\left(\dot{u}_{\epsilon}^{m}(t), \dot{u}_{\epsilon}^{m}(t)\right) \\
& \leq \quad C_{0}^{m}-a\left(u_{\epsilon}^{m}(t), \dot{u}_{\epsilon}^{m}(t)\right)+\left\langle f(t), \dot{u}_{\epsilon}^{m}(t)\right\rangle \\
& \quad+\int_{\Gamma_{f}}\left\{C_{n}+D_{n}\left|u_{\epsilon}^{m}(t)\right|^{m_{n}}\right\}\left|\dot{u}_{\epsilon}^{m}(t)\right| d s+\int_{0}^{t} a\left(\dot{u}_{\epsilon}^{m}(\alpha), \dot{u}_{\epsilon}^{m}(\alpha)\right) d \alpha \\
& \quad-\int_{0}^{t}\left\langle\dot{f}(\alpha), \dot{u}_{\epsilon}^{m}(\alpha)\right\rangle d \alpha+E_{n} \int_{0}^{t} \int_{\Gamma_{f}}\left\{1+\left|u_{\epsilon}^{m}(\alpha)\right|^{m_{n}-1}\right\}\left|\dot{u}_{\epsilon}^{m}(\alpha)\right|^{2} d s d \alpha \\
& \quad+E_{T} \int_{0}^{t} \int_{\Gamma_{f}}\left\{1+\left|u_{\epsilon}^{m}(\alpha)\right|^{m_{T}-1}\right\}\left|\dot{u}_{\epsilon}^{m}(\alpha)\right| M_{\epsilon}\left(\dot{u}_{\epsilon}^{m}(\alpha)\right) d s d \alpha,
\end{aligned}
$$


where $C_{0}^{m}$ is a bounded sequence of constants depending on $u_{0}^{m}, v_{0}^{m}$. Bearing in mind that $M_{\epsilon}(v) \leq \mu_{0}|v|$ we deduce that

$$
\begin{aligned}
& \int_{0}^{t} \int_{\Gamma_{f}}\left|u_{\epsilon}^{m}(\alpha)\right|^{m_{T}-1}\left|\dot{u}_{\epsilon}^{m}(\alpha)\right| M_{\epsilon}\left(\dot{u}_{\epsilon}^{m}(\alpha)\right) d s d \alpha \\
& \leq \int_{0}^{t}\left\|u_{\epsilon}^{m}(\alpha)\right\|_{L^{p}(\Gamma)}^{p-2}\left\|\dot{u}_{\epsilon}^{m}(\alpha)\right\|_{L^{p}(\Gamma)}^{2} d \alpha \leq C\left\|u_{\epsilon}^{m}\right\|_{L^{\infty}(0, T, V)}^{p-2}\left\|\dot{u}_{\epsilon}^{m}\right\|_{L^{2}(0, T, V)}^{2} .
\end{aligned}
$$

One can also deduce that

$$
\begin{aligned}
\int_{\Gamma_{f}}\left|u_{\epsilon}^{m}(t)\right|^{m_{n}}\left|\dot{u}_{\epsilon}^{m}(t)\right| d s & \leq\left\|u_{\epsilon}^{m}(t)\right\|_{L^{p}(\Gamma)}^{p-1}\left\|\dot{u}_{\epsilon}^{m}(t)\right\|_{L^{p}(\Gamma)} \\
& \leq C\left\|u_{\epsilon}^{m}\right\|_{L^{\infty}(0, T, V)}^{p-1}\left\|\dot{u}_{\epsilon}^{m}(t)\right\|_{V} .
\end{aligned}
$$

Using the above inequalities from (41), we get

$$
\begin{aligned}
\int_{0}^{t}\left|\ddot{u}_{\epsilon}^{m}(s)\right|^{2} d s+ & \frac{\eta}{2} d\left\|\dot{u}_{\epsilon}^{m}(t)\right\|_{V}^{2} \leq C\left\{1+\left[1+\left\|u_{\epsilon}^{m}\right\|_{L^{\infty}(0, T, V)}^{p-1}+\|f\|_{L^{\infty}\left(0, T, V^{\prime}\right)}\right]\left\|\dot{u}_{\epsilon}^{m}(t)\right\|_{V}\right. \\
& \left.+\left[\left\|u_{\epsilon}^{m}\right\|_{L^{\infty}(0, T, V)}^{p-2}+\left\|\dot{u}_{\epsilon}^{m}\right\|_{L^{2}(0, T, V)}+\|\dot{f}\|_{L^{2}(0, T, V)}\right]\left\|\dot{u}_{\epsilon}^{m}\right\|_{L^{2}(0, T, V)}\right\} .
\end{aligned}
$$

Using this last inequality and (39), we obtain that

$$
\dot{u}_{\epsilon}^{m} \text { is bounded in } L^{\infty}(0, T, V), \quad \ddot{u}_{\epsilon}^{m} \text { is bounded in } L^{2}(0, T, H) .
$$

From (39) and (42) it follows that there exists $u \in W^{1, \infty}(0, T, V) \cap W^{2,2}(0, T, H)$ and a subsequence of $u_{\epsilon}^{m}$, again denoted $u_{\epsilon}^{m}$, such that $u_{\epsilon}^{m} \rightarrow u$ weak $\star$ in $L^{\infty}(0, T, V), \dot{u}_{\epsilon}^{m} \rightarrow \dot{u}$ weak $\star$ in $L^{\infty}(0, T, V)$, and $\ddot{u}_{\epsilon}^{m} \rightarrow \ddot{u}$ weakly in $L^{2}(0, T, H)$ when $m \rightarrow \infty$ and $\epsilon \rightarrow 0$. This implies (30) and, if we denote by $Q=] 0, T\left[\times \Omega\right.$, we obtain that $u_{\epsilon}^{m} \rightarrow u$ and $\dot{u}_{\epsilon}^{m} \rightarrow \dot{u}$ weakly in $\left[H^{1}(Q)\right]^{N}$. Since $\left[H^{1}(Q)\right]^{N} \subset\left[L^{2}(Q)\right]^{N}=L^{2}(0, T, H)$, with compact injection we get

$$
u_{\epsilon}^{m} \rightarrow u, \quad \dot{u}_{\epsilon}^{m} \rightarrow \dot{u}, \quad \text { strongly in } L^{2}(0, T, H) .
$$

Let $w \in L^{2}(0, T, V)$ be fixed and let $w^{m} \in L^{2}\left(0, T, V_{m}\right)$ be such that $w^{m} \rightarrow w$ strongly in $L^{2}(0, T, V)$. If we put $v=w^{m}(t)$ in (36) after integrating from 0 to $T$ and after some algebra, we get

$$
\begin{aligned}
\int_{0}^{T}\left(\ddot{u}_{\epsilon}^{m}(t), w^{m}(t)\right) d t+\frac{1}{2}\left|v_{0}^{m}\right|^{2} & +\int_{0}^{T} a\left(u_{\epsilon}^{m}(t), w^{m}(t)\right) d t+\frac{1}{2} a\left(u_{0}^{m}, u_{0}^{m}\right) \\
+\eta \int_{0}^{T} c\left(\dot{u}_{\epsilon}^{m}(t), w^{m}(t)\right) d t & +\int_{0}^{T}\left[j\left(u_{\epsilon}^{m}(t), \dot{u}_{\epsilon}^{m}(t), w^{m}(t)\right)-j\left(u_{\epsilon}^{m}(t), \dot{u}_{\epsilon}^{m}(t), \dot{u}_{\epsilon}^{m}(t)\right)\right] d t \\
& +2 C T \epsilon\left(1+\left\|u_{\epsilon}^{m}\right\|_{L^{\infty}(0, T, V)}^{p-1}\right)
\end{aligned}
$$

Let us now prove that

$$
\int_{0}^{T} j\left(u_{\epsilon}^{m}(t), \dot{u}_{\epsilon}^{m}(t), \dot{u}_{\epsilon}^{m}(t)\right) d t \rightarrow \int_{0}^{T} j(u(t), \dot{u}(t), \dot{u}(t)) d t
$$


when $m \rightarrow \infty$ and $\epsilon \rightarrow 0$. Indeed, after some algebra we get

$$
\begin{aligned}
\mid j\left(u_{\epsilon}^{m}(t), \dot{u}_{\epsilon}^{m}(t), \dot{u}_{\epsilon}^{m}(t)\right)- & j(u(t), \dot{u}(t), \dot{u}(t)) \mid \\
\leq C\{(1+ & \left.\|u(t)\|_{L^{p+1}(\Gamma)}^{p-1}\right)\left\|\dot{u}(t)-\dot{u}_{\epsilon}^{m}(t)\right\|_{L^{p_{1}}(\Gamma)}\|\dot{u}(t)\|_{L^{p+1}(\Gamma)} \\
& +\left(1+\|u(t)\|_{L^{p}(\Gamma)}^{p-1}\right)\left\|\dot{u}(t)-\dot{u}_{\epsilon}^{m}(t)\right\|_{L^{p}(\Gamma)} \\
& \left.+\left(1+\|u(t)\|_{L^{p}(\Gamma)}^{p-2}+\left\|u_{\epsilon}^{m}(t)\right\|_{L^{p}(\Gamma)}^{p-2}\right)\left\|u(t)-u_{\epsilon}^{m}(t)\right\|_{L^{p}(\Gamma)}\|\dot{u}(t)\|_{L^{p}(\Gamma)}\right\}
\end{aligned}
$$

and we use Lemma 5.1 with

$$
\beta=\frac{3(p-1)+2}{2(p+1)}<1 \quad \text { if } N=3
$$

and

$$
\beta \in] \frac{p}{p+1}, 1[\text { if } N=2
$$

to deduce

$$
\left\|\dot{u}(t)-\dot{u}_{\epsilon}^{m}(t)\right\|_{L^{p+1}(\Gamma)} \leq C\left|\dot{u}(t)-\dot{u}_{\epsilon}^{m}(t)\right|^{1-\beta}\left(\|\dot{u}\|_{L^{\infty}(0, T, V)}+\left\|\dot{u}_{\epsilon}^{m}\right\|_{L^{\infty}(0, T, V)}\right)^{\beta} .
$$

From these last two inequalities, we obtain

$$
\begin{aligned}
& \int_{0}^{T}\left|j\left(u_{\epsilon}^{m}(t), \dot{u}_{\epsilon}^{m}(t), \dot{u}_{\epsilon}^{m}(t)\right)-j(u(t), \dot{u}(t), \dot{u}(t))\right| d t \\
& \quad \leq C\left\{\left\|\dot{u}-\dot{u}_{\epsilon}^{m}\right\|_{L^{2}(0, T, H)}^{1-\beta}+\left\|u-u_{\epsilon}^{m}\right\|_{L^{2}(0, T, H)}^{1-\beta}\right\}
\end{aligned}
$$

and using (43) we get (45). We can now pass to the limit when $\epsilon \rightarrow 0$ and $m \rightarrow+\infty$ in (44) to deduce (29).

6. Viscosity in the one-dimensional problem. Let us consider in this section a viscous perturbation of the problem (1)-(6). Let $\eta>0$ be a "small" viscosity parameter, and we associate with (1)-(6) the following initial and boundary value problem: find $v^{\eta}, \tau^{\eta}:[0, T] \times \Omega \rightarrow \mathbf{R}$ such that

$$
\begin{gathered}
\rho \dot{v}^{\eta}(t, x)=\partial_{x} \tau^{\eta}(t, x), \\
\dot{\tau}^{\eta}(t, x)=G \partial_{x} v^{\eta}(t, x)+\eta \bar{c}^{2} \partial_{x x} \tau^{\eta}(t, x),
\end{gathered}
$$

for all $(t, x) \in] 0, T] \times \Omega$,

$$
\begin{gathered}
v^{\eta}(t, H)=V, \\
\left|\tau^{\eta}(t, 0)\right| \leq \mu(0) S \quad \text { if } v^{\eta}(t, 0)=0, \\
\tau^{\eta}(t, 0)=S \mu\left(\left|v^{\eta}(t, 0)\right|\right) \frac{v^{\eta}(t, 0)}{\left|v^{\eta}(t, 0)\right|} \quad \text { if } v^{\eta}(t, 0) \neq 0
\end{gathered}
$$

for all $t \in] 0, T]$,

$$
v^{\eta}(0, x)=v_{0}(x), \quad \tau^{\eta}(0, x)=\tau_{0}(x) \quad \text { for all } x \in \Omega .
$$


This problem represents the one-dimensional dynamical shearing of an infinite linear visco-elastic slab that is in frictional contact with a rigid body. Indeed, if we consider the linear visco-elastic constitutive law given by (10), we have

$$
\tau^{\eta}=G \partial_{x} u^{\eta}+\eta \bar{G} \partial_{x} \dot{u}^{\eta}
$$

and then from the momentum balance law (46), we deduce $(47)$ with $\bar{c}=\sqrt{\bar{G}} / \rho$ and $v^{\eta}=\dot{u}^{\eta}$.

Having in mind that (46)-(51) has a unique smooth solution, $v^{\eta}, \tau^{\eta}$ (see Theorem 5.1 , we want to recover a solution $v, \tau$ of (1)-(6) as the limit as $\eta \rightarrow 0$. The following numerical simulations show that the solution of (1)-(6) selected by the above asymptotic method of vanishing viscosity is the solution that is selected by the perfect delay criterion. We are not able to give a proof of this last statement, which is still an open problem. However, the following conjecture, can be stated: in the elastic case, the solution chosen by the perfect delay convention is the one obtained from the solutions of the problem with viscosity, when the viscosity tends to zero.

In order to illustrate our purpose, we consider $\mu$ as a piecewise linear function, as follows:

$$
\mu(v)=\mu_{s}-\frac{\mu_{s}-\mu_{d}}{\alpha_{1}} v \quad \text { if } v \leq \alpha_{1}, \quad \mu(v)=\mu_{d} \quad \text { if } v \geq \alpha_{1}
$$

where $\mu_{s}>\mu_{d}$ are the static and dynamic friction coefficients. We have chosen the constants $\mu_{s}, \mu_{d}$, and $\alpha_{1}$ such that the function $g$, given by (8), is decreasing on $\left[0, \alpha_{1}\right]$. We performed a cycle of loading, i.e., we have chosen $v_{0}, \tau_{0}$ such that $t \rightarrow \beta(t)$ is first increasing and then decreasing. In this way, we can see the whole path followed by the system in a hysteresis or a stick-slip-stick sequence.

In the numerical simulations plotted below, we have used a finite difference method. In the interior of the domain, an explicit scheme was used. A CFL condition for the hyperbolic part and a stability condition for the parabolic part of the linear operator are required in this scheme. For the boundary condition at $x=0$, we have used a Newton method to solve a nonlinear equation, deduced from the friction law after an integration on the characteristic lines.

In Fig. 2 the time evolution of the slip rate in the elastic case, i.e., $t \rightarrow v(t, 0)$ (with $v$ given by the perfect delay criterion) and in the visco-elastic case, i.e., $t \rightarrow v^{\eta}(t, 0)$ for different values of $\eta$ are plotted. Note that when the viscosity is vanishing, $v^{\eta}$ approaches the solution $v$ chosen by the perfect delay criterion in the elastic case. As was shown in Sec. 2 (see Fig. 1 for a generic representation), the elastic solution has two shocks during a cycle. Indeed, the first one is from 0 to $\alpha_{2}$ during the loading and the second is from $\alpha_{1}$ to 0 during the unloading. One can remark that the viscosity has a smoothing effect on these two shocks. 


$$
\begin{aligned}
& \text { - no viscosity } \quad \cdots \cdots \cdot \eta=0.005 \\
& \cdots \eta=0.01 \quad \cdots \cdot \cdot \eta=0.001
\end{aligned}
$$

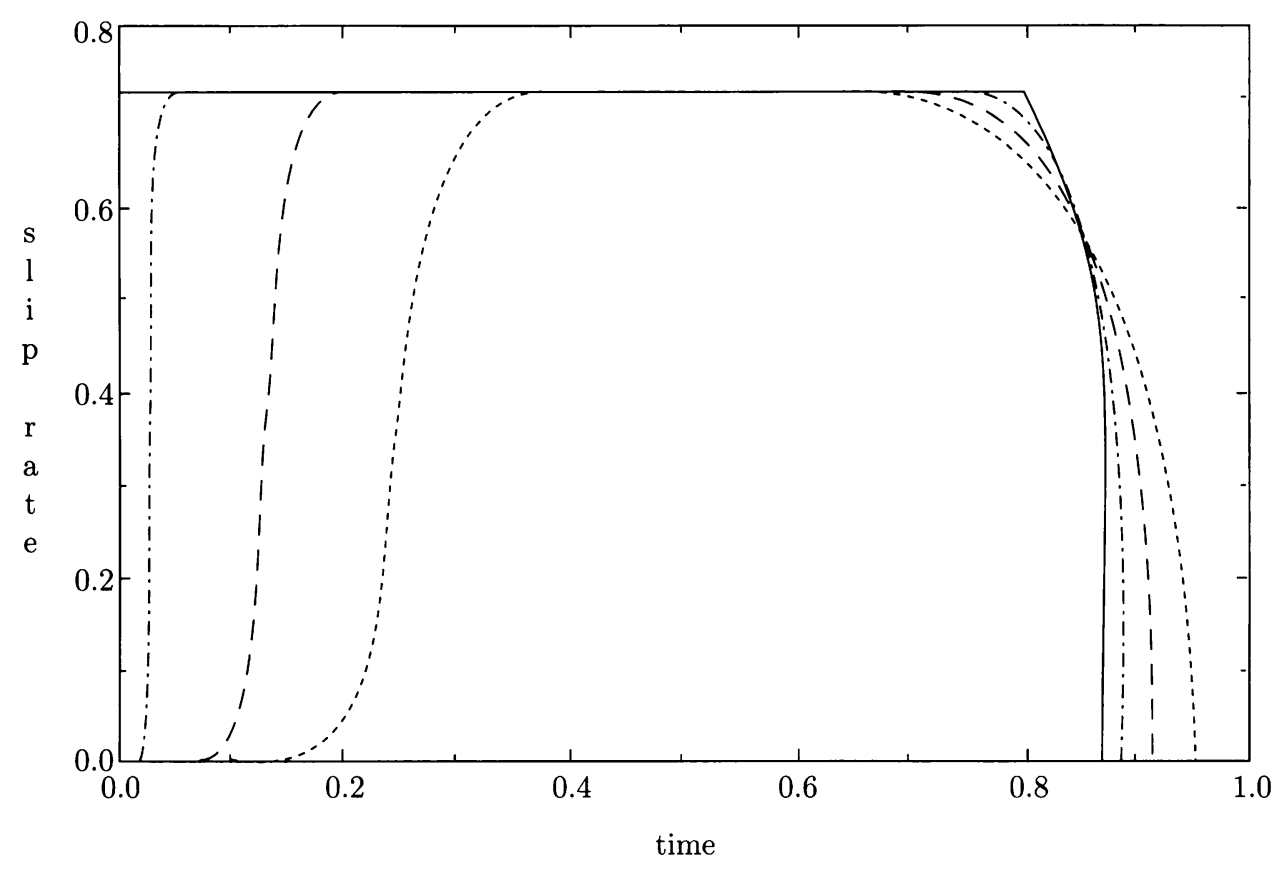

FIG. 2. The time evolution of the slip rate in the elastic case $t \rightarrow$ $v(t, 0)$ discriminated by the perfect delay convention (the solid line) and in the visco-elastic case $t \rightarrow v^{\eta}(t, 0)$ for $\eta=0.01, \eta=0.005, \eta=$ 0.001 (the dashed lines). Note the convergence of the viscous solution to the solution of the elastic problem and the smoothing effect of the viscosity on the shocks.

\section{REFERENCES}

[1] M. Campillo, I. R. Ionescu, J.-C. Paumier, and Y. Renard, On the dynamic sliding with friction of a rigid block and of an infinite elastic slab, Physics of the Earth and Planetary Interiors 96, 15-23 (1996)

[2] G. Duvaut and J. L. Lions, Inequalities in Mechanics and Physics, Springer-Verlag, Berlin-New York, 1976

[3] J. H. Dieterich, A constitutive law for rate of earthquake production and its application to earthquake clustering, J. Geophys. Res. 99, No. B2, 2601-2618 (1994)

[4] P. Favreau, I. R. Ionescu, and M. Campillo, On the dynamic sliding with rate and state dependent friction laws, Geophysical Journal 139, 671-678 (1999)

[5] I. R. Ionescu and J.-C. Paumier, Dynamic stick-slip motions with sliding velocity-dependent friction, Comptes Rendus Acad. Sci. Paris. Sér. I Math. 316, 121-125 (1993)

[6] I. R. Ionescu and J.-C. Paumier, On the contact problem with slip rate dependent friction in elastodynamics, European J. Mech. A Solids 13, 555-568 (1994)

[7] I. R. Ionescu and J.-C. Paumier, On the contact problem with slip displacement dependent friction in elastostatics, Internat. J. Engrg. Sci. 34, 471-491 (1996)

[8] J. Jarusek and C. Eck, Dynamic contact problems with small Coulomb friction for viscoelastic bodies. Existence of solutions, Math. Models Methods Appl. Sci. 9, 11-34 (1999)

[9] K. L. Kuttler, Dynamic friction contact problems for general normal and friction laws, Nonlinear Analysis 28, 559-575 (1997) 
[10] K. L. Kuttler, Y. Renard, and M. Schillor, Models and simulations of dynamic frictional contact of a beam, Comput. Meth. Appl. Mech. Engrg. 177, 257-272 (1999)

[11] K. L. Kuttler and M. Schillor, Set-valued pseudomonotone maps and degenerate evolution inclusions, Communications in Contemporary Mathematics 1, 87-123 (1999)

[12] J. A. C. Martins and J. T. Oden, Existence and uniqueness results for dynamic contact problems with nonlinear normal and friction interface laws, Nonlinear Analysis 11, 407-428 (1987)

[13] J. T. Oden and J. A. C. Martins, Models and computational methods for dynamic friction phenomena, Comput. Math. Appl. Mech. Engrg. 52, 527-634 (1985)

[14] G. Perrin, J. R. Rice, and G. Zheng, Self-healing slip pulse on a frictional surface, Journal of the Mechanics and Physics of Solids 43, 1461-1495 (1995)

[15] T. Poston and I. Stewart, Catastrophe Theory and its Applications, Pitman, London, 1978

[16] J. R. Rice and A. L. Ruina, Stability of steady frictional slipping, Journal of Applied Mechanics 50, 343-349 (1983)

[17] Y. Renard, Perturbation singulière d'un problème de frottement sec non monotone, Comptes Rendus Acad. Sci. Paris, Sér. I. Math. 326, 131-136 (1998)

[18] Y. Renard, Singular perturbation approach to an elastic dry friction problem with nonmonotone coefficient, Quart. Appl. Math. 58, 303-324 (2000)

[19] A. L. Ruina, Slip instabilities and state variable friction laws, J. Geophysics Res. 88, B12, 10359$10370(1983)$

[20] C. H. Scholtz, The Mechanics of Earthquakes and Faulting, Cambridge Univ. Press, Cambridge, 1990 